\title{
Which is the single most important factor in deciding the management and determining the prognosis of head and neck cancer?
}

\author{
Alfio Ferlito • Robert P. Takes • Alessandra Rinaldo • \\ Kenneth O. Devaney
}

Received: 15 September 2009 / Accepted: 22 September 2009 / Published online: 13 October 2009

(C) Springer-Verlag 2009

The literature is full of references to the identification of different factors that may be helpful in deciding the treatment and determining the prognosis in patients with head and neck tumors. However, which factor is the most important and decisive in the treatment and prognosis?

It is common practice for the clinician to speak with and inform the patient after receiving the report of the histopathological examination which, often confusingly for the patient, could be described as "positive" or "negative". This histologic diagnosis, based on light microscopy, may be supported by immunohistochemical, ultrastructural and molecular investigations that improve the accuracy of categorization of the specific tumor type, and is the only specific diagnosis of the disease of the patient. Therefore, the role of the pathologist is very important. He or she is called upon to provide, what could be called, a qualitative diagnosis: for example, by labeling a neoplasm as a squamous cell carcinoma, verrucous squamous cell carcinoma, small cell neuroendocrine carcinoma, adenoid cystic carcinoma, melanoma, rhabdomyosarcoma, lymphoma and so on. It is up to the clinician and radiologist to produce a quantitative

\footnotetext{
A. Ferlito $(\bowtie) \cdot$ A. Rinaldo

Department of Surgical Sciences, ENT Clinic,

University of Udine, Policlinico Universitario,

Piazzale S. Maria della Misericordia, 33100 Udine, Italy

e-mail: a.ferlito@uniud.it

R. P. Takes

Department of Otolaryngology-Head and Neck Surgery,

Radboud University Nijmegen Medical Centre,

Nijmegen, The Netherlands

K. O. Devaney

Department of Pathology,

Allegiance Health, Jackson, MI, USA
}

diagnosis of the lesion and possibly a differential diagnosis. The combination of clinical information complementary to the histopathological examination is often helpful to establish the precise diagnosis. An example may be the differentiation between a squamous cell cancer of the skin versus a keratoacanthoma. Another example may be the difference between a clinically infiltrating tumor and a histopathological report of dysplasia: a disparity, which is most often the result of a sampling error. The product of these diagnoses will give rise to a more detailed understanding of the disease, resulting in more effective treatment and a more reliable prognosis.

For primary small cell neuroendocrine carcinoma of the head and neck, an extensive and systematic workup is mandatory for therapeutic and prognostic reasons. Such a workup might be expected to include endoscopy of the upper aerodigestive tract, imaging with CT and/or MRI of the head and neck, chest, brain, upper abdomen, pulmonary evaluation, screening for distant metastasis and laboratory investigations.

In contrast, the evaluation and tumor staging in verrucous squamous cell carcinoma is completely different from that for small cell neuroendocrine carcinoma. Verrucous squamous cell carcinoma is a tumor that is locally aggressive and non-metastasizing, which means that extensive tumor staging or evaluation is not indicated. Verrucous squamous cell carcinoma may be cured by conventional or endoscopic laser surgery and neck dissection is not indicated, as this tumor has so far never metastasized to cervical lymph nodes or to any other organs. The common clinical finding of enlarged and tender lymph nodes draining verrucous squamous cell carcinoma may be misinterpreted as proof of metastatic disease. However, pathologic assessment of such lymph nodes consistently reveals only an inflammatory reaction. Radiotherapy is far less effective 
than surgery because this tumor, though not wholly radioresistant, is much less radiosensitive than conventional squamous cell carcinoma [1]. Patients receiving initial treatment with surgery enjoyed better survivals than did those treated with irradiation, especially for cases originating in the oral cavity, as suggested by the National Cancer Database records of 2,350 cases of verrucous squamous cell carcinoma of the head and neck [2]. This poor response to radiotherapy is in stark contrast to the usually good response to chemoradiotherapy of the previously described small cell neuroendocrine carcinoma.

In summary, the exact identification of the type of cancer enables clinicians to plan specific and individual tumor staging and treatment according to the oncotype involved. Moreover, to avoid mismanagement of the patient and complications, it is mandatory that the correct diagnosis be established prior to commencement of the definitive treatment. The choice of treatment is an important parameter not only influencing prognosis, but also of at least equal importance the patient's subsequent quality of life. Obviously, long-time prognosis would also be superior if the most appropriate therapy was undertaken "first time" [3].

So, the decisions regarding the most suitable diagnostics and therapy are necessarily based on the histologic type of the tumor. The prognosis, obviously, depends largely on the different biological behaviors of the various oncotypes. Other factors such as anatomical extension (stage of the disease), presence of another malignancy, comorbidities, environmental aspects and previous treatment are of considerable prognostic significance, but the oncotype is the most important factor in deciding the treatment and determining the prognosis in head and neck cancer. The prognosis of different oncotypes differs enormously: it is excellent for verrucous squamous cell carcinoma, generally favorable for common squamous cell carcinoma and poor for small cell neuroendocrine carcinoma. More specifically, 5-year survival rates are $95 \%$ for verrucous squamous cell carcinoma, $90 \%$ for chondrosarcoma, $80 \%$ for mucoepidermoid carcinoma, $68 \%$ for squamous cell carcinoma, $68 \%$ for spindle cell carcinoma, $48 \%$ for carcinoid tumor, $46 \%$ for atypical carcinoid tumor, $20 \%$ for melanoma, $17.5 \%$ for basaloid squamous cell carcinoma and 5\% for small cell neuroendocrine carcinoma of the larynx, considering all stages of disease [4].

The presence of lymph node metastasis is usually considered the single most adverse important prognostic factor in head and neck cancer, but this is not always true, as for example in the case of a primary adenoid cystic carcinoma. For this tumor, distant metastases may be detected in the lung, liver, bones or brain without involvement of cervical lymph nodes.

The optimal management of a patient with cancer depends on many factors, including general health and patient preference, and it is largely governed by the stage of the disease at presentation. However, the oncotype is the single most important factor, which guides the management and prognosis of a neoplastic patient.

Most head and neck cancers that have to be treated in current practice will be squamous cell cancers; unfortunately, despite extensive research, no specific histopathological, biological or molecular factors have been identified within this histologic type to date that are able to significantly influence decision-making and prognostication. Recent research has for example identified molecular factors related to response to treatment, such as the presence of HPV in oropharyngeal cancer and RNA expression profiles related to the presence or absence of regional metastasis $[5,6]$. In future, the identification of molecular factors and profiles in head and neck squamous cell cancer may prove to be as important as the identification nowadays of the correct histopathological oncotype. However, for now, it is important to realize that there are many other histological types of head and neck cancer, all of which have their specific natural histories and commensurate diagnostic workup and therapeutic and prognostic implications that eclipse all other known factors.

\section{References}

1. Ferlito A, Rinaldo A, Mannarà GM (1998) Is primary radiotherapy an appropriate option for the treatment of verrucous carcinoma of the head and neck? J Laryngol Otol 112:132-139

2. Koch BB, Trask DK, Hoffman HT, Karnell LH, Robinson RA, Zhen W, Menck HR (2001) Commission on Cancer, American College of Surgeons, American Cancer Society. National survey of head and neck verrucous carcinoma: patterns of presentation, care, and outcome. Cancer 92:110-120

3. Ferlito A, Boccato P, Shaha AR, Carbone A, Noyek AM, Doglioni C, Bradley PJ, Rinaldo A (2001) The art of diagnosis in head and neck tumors. Acta Otolaryngol 121:324-328

4. Ferlito A, Bailey B, Rinaldo A (2009) Prognostic factors of laryngeal cancer. In: Fried MP, Ferlito A (eds) The Larynx, vol II, 3rd edn. Plural Publishing, San Diego, pp 699-709

5. Fakhry C, Westra WH, Li S, Cmelak A, Ridge JA, Pinto H, Forastiere A, Gillison ML (2008) Improved survival of patients with human papillomavirus-positive head and neck squamous cell carcinoma in a prospective clinical trial. J Natl Cancer Inst 100:261-269

6. Roepman P, Wessels LF, Kettelarij N, Kemmeren P, Miles AJ, Lijnzaad P, Tilanus MG, Koole R, Hordijk GJ, van der Vliet PC, Reinders MJ, Slootweg PJ, Holstege FC (2005) An expression profile for diagnosis of lymph node metastases from primary head and neck squamous cell carcinomas. Nat Genet 37:182-186 